\title{
First physics with ALICE: from p-p to heavy ions
}

\author{
Domenico ELIA* \\ INFN Sezione di Bari, Bari (Italy) \\ E-mail: Domenico.Elia@ba.infn.it
}

The ALICE experiment has been designed to measure the properties of strongly interacting matter created in heavy-ion collisions at LHC. The apparatus has several features, such as low $\mathrm{p}_{T}$ acceptance and powerful tracking over a broad momentum range, that make ALICE also an important contributor to the proton-proton LHC physics. In this respect it aims both at setting the baseline for the understanding of the heavy-ion data and exploring the new energy domain. After an introductory description of the status of the experiment, this paper deals with the ALICE physics potential in particular discussing the early $\mathrm{p}-\mathrm{p}$ and $\mathrm{Pb}-\mathrm{Pb}$ running scenarios and the corresponding physics programmes.

2008 Physics at LHC

September 29 - 4 October 2008

Split, Croatia

*Speaker.

${ }^{\dagger}$ On behalf of the ALICE Collaboration. 


\section{Introduction}

The Large Hadron Collider (LHC) at CERN is expected to provide first collisions within 2009, starting with the p-p system up to the top centre-of-mass energy $\sqrt{s}=14 \mathrm{TeV}$. In the first years of data taking $\mathrm{Pb}-\mathrm{Pb}$ collisions at $\sqrt{s_{N N}}=5.5 \mathrm{TeV}$ will also be delivered for about $10 \%$ of the effective machine time ( $10^{6}$ seconds). A Large Ion Collider Experiment (ALICE) is the LHC experiment specifically devoted to the physics of ultra-relativistic heavy-ion collisions and the study of the quark-gluon plasma (QGP) phase[1, 2]. However, some features of the experimental apparatus in terms of design and performance will allow ALICE to uniquely contribute to the p-p LHC physics as well[3]. In the next sections the status of the experiment and the perspectives for the first $p-p$ and $\mathrm{Pb}-\mathrm{Pb}$ data are illustrated and discussed.

\section{ALICE experiment at LHC}

The ALICE apparatus has been designed as a dedicated heavy-ion detector optimized to measure a large variety of observables in very high multiplicity environments (up to 4000 charged particles per unit of rapidity with performance checked up to 8000). It will be able to detect and identify hadrons, leptons and photons over a wide range of momenta. The whole detector, shown in Figure 1, consists of a central part $(|\eta|<0.9)$ to detect hadrons, electrons and photons, a forward spectrometer to measure muons and additional smaller forward detectors for event characterization and triggering. A detailed description of the apparatus can be found in [2, 4].

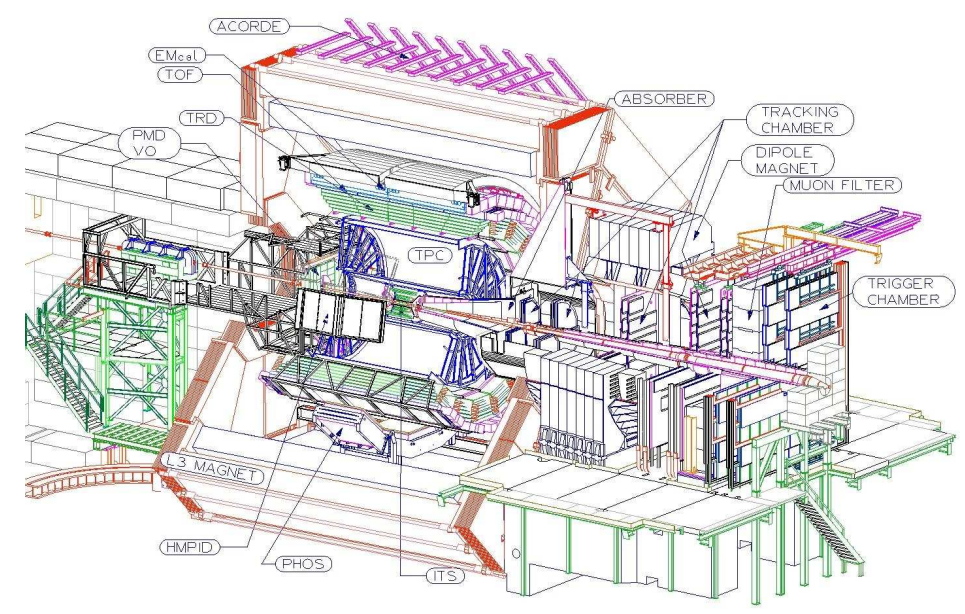

Figure 1: General view of the ALICE detector.

The Silicon Pixel Detector (SPD), the Time Projection Chamber (TPC) and the V0 detector will play a key role for the first data: as most of the other sub-systems, they are fully installed and commissioned[5]. The SPD is the innermost element of the ALICE Inner tracking System (ITS), consisting of two layers of hybrid silicon pixels surrounding the beam pipe at 3.9 and 7.6 $\mathrm{cm}$ average radii with a total of $\approx 10^{7}$ pixel cells. It features a very low material budget $(\approx 1 \%$ per layer), a detection efficiency above $99 \%$, a spatial resolution of $\approx 12 \mu \mathrm{m}$ in the bending plane and a prompt signal as input to the level 0 trigger[4]. The SPD will allow the measurement of the charged particle multiplicity and pseudo-rapidity density distributions with a low momentum cut-off $(\approx 35$ 
$\mathrm{MeV} / c$ at $0.5 \mathrm{~T}$ field) up to $|\eta| \approx 2$. Furthermore, the so-called "Fast-OR" digital pulses coming from each of the 1200 SPD chips (indicating at least one pixel hit within the chip) will contribute to the minimum bias trigger and allow triggering on high multiplicity events[3,6].

The TPC has a cylindrical sensitive volume with radii between 85 and $250 \mathrm{~cm}$ for a length of $500 \mathrm{~cm}$ : it is the largest in the world and has been optimized for high track densities. It can track particles in $|\eta|<0.9$ with efficiency above $90 \%$, excellent momentum resolution up to $100 \mathrm{GeV} / c$ and particle identification up to $1 \mathrm{GeV} / c$. The $\mathrm{V} 0$ detector consists of a pair of tiled scintilator disks on either side of the interaction point: it will provide the minimum bias trigger (combined with the SPD Fast-OR) togheter with beam-gas background rejection and luminosity information[3].

\section{First proton-proton run}

While designed to deal with heavy-ion collisions and study the QGP properties, ALICE interest for the p-p LHC programme goes beyond the need to provide reference data for $\mathrm{Pb}-\mathrm{Pb}$. Its unique detection capabilities (tracking to a very low $\mathrm{p}_{T}$, excellent particle identification, very low mass tracking system) will allow to address a number of important studies within the p-p physics. The first data taking scenario, starting from the year 2009, is based on a 10 or $14 \mathrm{TeV}$ p-p run at nominal luminosity $\mathscr{L} \simeq 3 \times 10^{30} \mathrm{~cm}^{-2} \mathrm{~s}^{-1}$. Running for $10^{7} \mathrm{~s}$ with a geometrical cross section $\sigma=0.07 \mathrm{~b}$, in the first year of data taking $2 \times 10^{12}$ collisions will take place and $10^{9}$ minimum bias collisions will be collected. At the start-up some collisions at $900 \mathrm{GeV}$ could be delivered: this would be very useful in connection to the existing measurements and then to the systematics issues. The efficient minimum bias event trigger will allow ALICE to perform inclusive studies aimed at QCD measurements as those mentioned in the following.

In the left panel of Figure 2 the integral number of events above a given multiplicity, for different sample sizes of PYTHIA non-single diffractive (NSD) events, is shown.
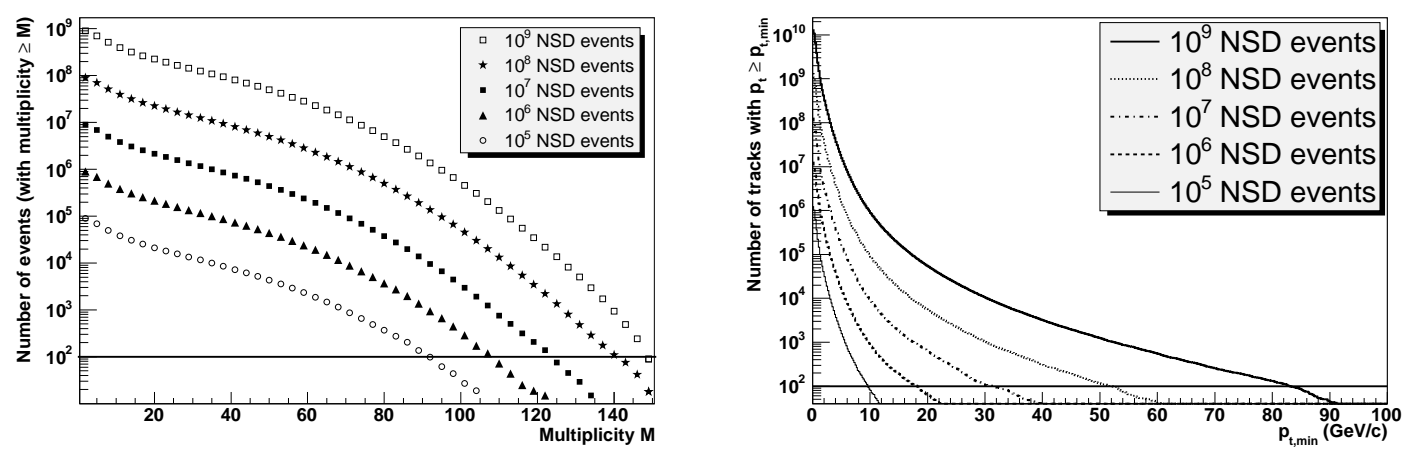

Figure 2: Left panel: number of events over a given multiplicity in $|\eta|<0.9$ and for different statistics of NSD simulated p-p events. Right panel: number of charged particles with $|\eta|<0.9$ above a given $\mathrm{p}_{T}$.

With a statistics of 20,000 to 40,000 events (first few days, with a multiplicity reach up to $\approx$ 5 times the mean multiplicity) a measurement of the charged particle pseudo-rapidity density and multiplicity distributions can be properly performed[7]. As those observables correspond to basic properties of the collisions in the new energy domain at LHC, their knowledge will allow to correctly configure the Monte Carlo generators. Moreover, the measurement of the charged-particle 
pseudorapidity density in the central rapidity region will extend the existing energy dependence pattern. Besides these very first measurements, $\mathrm{p}_{T}$ spectra of both all charged and identified particles (the ALICE $\mathrm{p}_{T}$ reach is shown in the right panel of Figure 2), baryon number transport and strangeness production $\left(\mathrm{K}^{ \pm}, \mathrm{K}_{s}^{0}, \Lambda\right.$ and $\left.\bar{\Lambda}\right)$ analyses will also be carried out within the p-p first physics programme.

\section{Early heavy-ion run}

The first heavy-ion run is scheduled for 5.5 A TeV Pb-Pb collisions at reduced luminosity $\mathscr{L} \simeq 5 \times 10^{25} \mathrm{~cm}^{-2} \mathrm{~s}^{-1}$, corresponding to $1 / 20$ of the design luminosity. Running for $10^{6} \mathrm{~s}$ should be enough to collect $10^{7}$ minimum bias and another $10^{7}$ central (5\%) collisions. Since following the first p-p run, for this data taking a fully commissioned detector is expected: in particular alignment and calibrations will be available from the previuosly collected cosmics and p-p samples. Data quality and statistics should already allow with this pilot run to explore a quite rich physics spectrum. The initial $10^{5}$ events will provide information about global event properties such us multiplicity, pseudo-rapidity density and elliptical flow. Indeed the very first measurement in the ALICE heavy-ion physics programme will be the charged particle multiplicity density at mid-rapidity, followed by its behaviour along the $\eta$ range covered by the apparatus. Figure 3 shows a collection of multiplicity results from heavy-ion and $p-\bar{p}$ collisions, where the A-A data are rescaled by the number of nucleons participating in the collision[2].

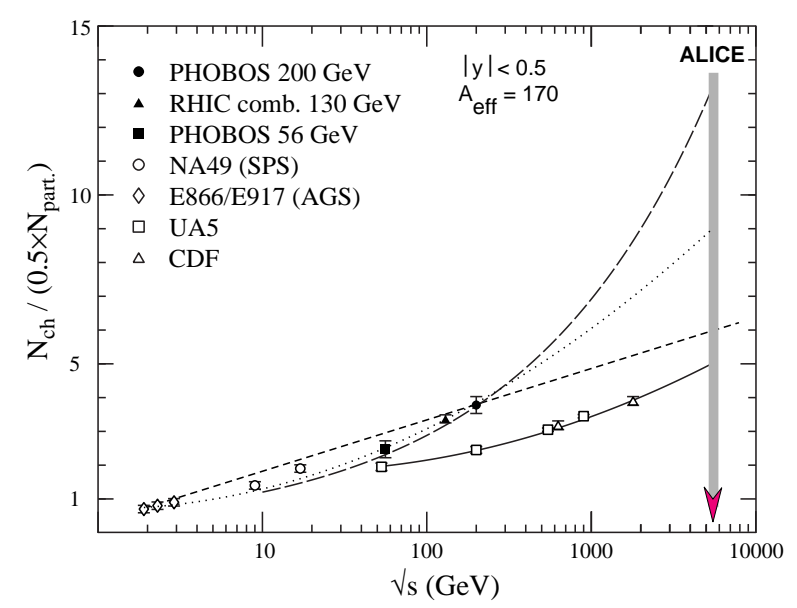

Figure 3: Charged particle rapidity density per participant pair as a function of centre-of-mass energy for A-A and p-p collisions. Long dashed line is an extrapolation to LHC energies based on the saturation model.

When extrapolating from existing data to LHC energies there is a striking difference between the results obtained applaying a saturation model[8] (long dashed line) or a fit in $\ln ^{2} \sqrt{s}$ (dashed line). The expected values for the $\mathrm{d} N_{c h} / \mathrm{d} \eta$ plateau level range from 1200 to 2600 (for most central $5 \%$ collisions), substantially lower than the ALICE design value. The measurement will challenge the models currently describing particle production in nuclear collisions up to the RHIC energies.

With a factor 10 more statistics $\left(10^{6}\right.$ events) particle spectra, resonances, differential flow and interferometry analyses will be reasonably accessible. The copious multiplicity of produced particles will allow to address essential measurements such as the particle composition and the 
transverse momentum distributions of identified particles. As an example, ALICE will have reconstruction rates of $13,0.1$ and 0.01 per event for the $\Lambda, \Xi$ and $\Omega$ hyperons respectively. The excellent performance in terms of tracking, vertexing and particle identification capabilities will be key factors: in Figure 4 displaced vertices from a cascade decay and an axample of $\pi$ p invariant mass distribution with the $\Lambda$ peak in simulated central $\mathrm{Pb}-\mathrm{Pb}$ collisions are shown.
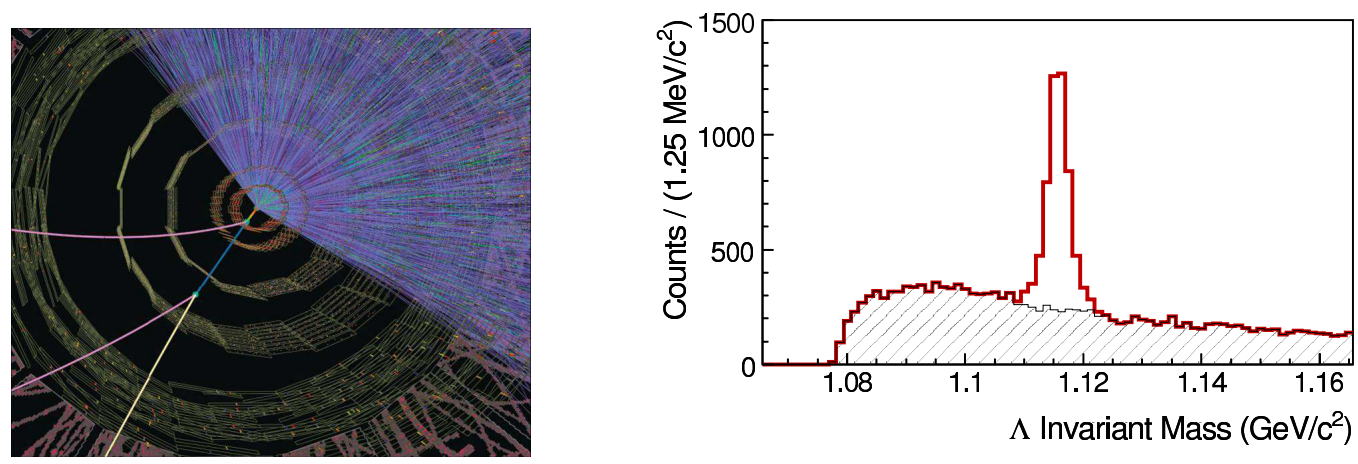

Figure 4: Left panel: fraction of $\mathrm{Pb}-\mathrm{Pb}$ event in the ITS with displaced vertices from a cascade decay. Right panel: $\pi$ p invariant mass distribution with the $\Lambda$ peak in central $\mathrm{Pb}-\mathrm{Pb}$ collisions.

Statistically relevant samples from as little as $10^{6}$ events will provide freeze-out temperature and collective motion of the particle emitting source and allow to verify the "hadrochemistry" thermal models which have successful described hadron production up to RHIC energies[9]. In addition, bulk properties of the medium (jet quenching), heavy-flavours and charmonia production will also be achieved with a full sample of $10^{7}$ events from such first $\mathrm{Pb}-\mathrm{Pb}$ pilot run[3].

\section{Summary and outlook}

ALICE is going to collect first $\mathrm{p}-\mathrm{p}$ and $\mathrm{Pb}-\mathrm{Pb}$ data starting from the second half of the year 2009. From the first few days of data taking a global characterization of the p-p collisions will be accessible. The running conditions and the expected detector performance will allow to address both in the first proton run and the following short pilot heavy-ion run an already quite rich and uniquely interesting physics programme.

\section{References}

[1] ALICE Technical Proposal, CERN/LHCC 95-71 (1995).

[2] F. Carminati et al., ALICE: Physics Performance Report Volume I, J. Phys G 30 (2004) 1517.

[3] B. Alessandro et al., ALICE: Physics Performance Report Volume II, J. Phys G 32 (2006) 1295.

[4] K. Aamodt et al., JINST 3 (2008) S08002.

[5] J. Shukraft et al., these Proceedings.

[6] J. Conrad et al., ALICE-INT-2005-025 (2005).

[7] J. F. Grosse-Oetringhaus et al., these Proceedings.

[8] k. J. Eskola et al., Nucl. Phys. B570 (2000), 379.

[9] A. Andronic et al., Nucl. Phys. A772 (2006), 167. 